Acta Theriologica 44 (3): 321-327, 1999.

PL ISSN 0001-7051

\title{
Age estimation in Iberian wildcats Felis silvestris, by canine tooth sections
}

\author{
Rosa GARCÍA-PEREA and Rocío A. BAQUERO
}

\begin{abstract}
García-Perea R. and Baquero R. A. 1999. Age estimation in Iberian wildcats Felis silvestris, by canine tooth sections. Acta Theriologica 44: 321-327.

We have applied counting of annual cementum lines in canine root to estimate the age of 165 Iberian wildcats Felis silvestris Schreber, 1775. Results indicate that (1) closure of apical foramen of canine root occurs from 6 to 18 months of age, (2) cementum lines are formed in autumn-winter, (3) the first autumn-winter, either a non-continuous line, a continuous "kitten line" or no line can be formed, depending on individual variation in timing of closure of apical foramen, (4) since $7 \%$ of wildcats form a kitten line, it needs to be identified in order to avoid an incorrect counting, (5) the first continuous line useful for counting is formed during the second autumn-winter, when animals are 21 months old. For Iberian wildcats, age in years can be estimated by adding 1 to the number of continuous lines. For a more accurate estimation, we propose a formula including months from birth to first line formation, number of annual lines, and months from last line formation to death $(A=9 m+L y+M m)$.
\end{abstract}

Museo Nacional de Ciencias Naturales, C/ J. Gutierrez Abascal 2, Madrid 28006, Spain, e-mail: mcng3100@mncn.csic.es

Key words: Felis silvestris, age estimation, Iberian Peninsula, tooth development

\section{Introduction}

Age is an essential factor to conduct certain studies on morphology, biology and ecology of wild populations, since it allows to describe ontogenetic variation, to establish timing of biological processes, and to calculate demographic parameters in pcpulations, among others. However, we usually have no information about the exact age of animals captured in the wild, and it has been necessary to develop methods for age estimation.

Counting of annual cementum lines in canine root is a method widely applied on carnvores and generally agreed as an accurate way to estimate the age in years (Klevezal and Kleinenberg 1967, Morris 1972, Grue and Jensen 1979, Johnson et al. 1981). This method has been successfully applied on both, wild and domestic felids, eg lyaxes (Crowe 1972, Kvam 1984, Zapata et al. 1997) and domestic cat (Grue and Jensen 1979).

Usually, age in years is estimated by adding 1 to the number of lines (eg Grue and Jensen 1979), but it is possible to attain a more accurate estimate by considering birth and death parameters (García-Perea 1991). 
Although processes leading to line formation and factors governing it are similar for carnivores living in temperate regions, there are some differences among species, related to the birth date and the timing of the developmental processes associated to the teeth. For this reason, the general rule of adding 1 to the number of lines is not always true (see, eg, Zapata et al. 1995), and the protocol needs to be adapted for each species, sometimes even for different populations of the same species (Grue and Jensen 1979).

In this article, we provide information about the parameters necessary to estimate age in Iberian populations of Felis silvestris Schreber, 1775, by counting the annual cementum lines in canine teeth.

\section{Material and methods}

We have examined 165 skulls of Felis silvestris from different areas of the Iberian Peninsula. These materials are kept in the following institutions: UZA collection/Museo Nacional de Ciencias Naturales (Madrid, Spain), Estación Biológica de Doñana (Seville, Spain), and several Spanish private collections (see Acknowledgements). Specimens were identified as Felis silvestris by Schauenberg's index (1969), expressing the quotient between total length and cranial capacity of skull. $F$. silvestris shows values equal to or under 2.75 , and $F$. catus over that figure. Total length of skull has been measured with a digital caliper to the nearest $0.02 \mathrm{~mm}$, and cranial capacity with small shots $2 \mathrm{~mm}$ in diameter and a test tube $1 \mathrm{cc}$ of accuracy.

Specimens were selected from a larger sample by having permanent teeth and information about the month or season of death. Cementum development starts in the root simultaneously to permanent teeth eruption, resulting in both closure of apical foramen and deposit of annual layers of cementum (Grue and Jensen 1979). Therefore, although most specimens of the sample had a closed apical foramen, some specimens with different stages of closure of the foramen were selected in order to establish the timing of that process.

One lower canine was removed from each specimen. A piece $7-10 \mathrm{~mm}$ long was sectioned from the tip of each root. These pieces were decalcified, sectioned on a freezing-microtome to $14 \mu$ thickness, stained with Mayer's haemalun and mounted (Grue and Jensen 1979). Slides were examined under 100x magnification. Each annual deposit of cementum consists of two zones of low and high density, observed as light and dark lines respectively under microscope. Dark lines are those used for age estimation.

Based on the protocol followed by Grue and Jensen (1979), information necessary for age estimation by this method are: period of closure of apical foramen, period of dark line formation, and age of formation of the first dark line.

In order to determine the period of line formation, specimens showing a "multiple line" under development were not considered. Each multiple line consists of a primary line, generated during the usual period of line formation, and one or more secondary lines, formed at different times of the year (Grue and Jensen 1979) related to periods of physical stress such as the rut in ungulates (Reimers and Nordby 1968).

To test for seasonal differences in line formation, we have compared values observed in the sample for the periods autumn-winter versus spring-summer, based on information existing on other carnivores from temperate regions, forming in general the lines in autumn-winter (Morris 1972, Grue and Jensen 1979).

To find out whether wildcats form or not a line during the first autumn-winter, we examined the youngest specimens of the sample. Age of these individuals was estimated by assuming April to be the month of birth (Schauenberg 1974, Stahl et al. 1988), and by using cranial criteria (García-Perea et al. 1996). 


\section{Results}

\section{Closure of apical foramen}

Specimens presenting an open apical foramen in the canine root $(n=6)$, or some stage of closure of the foramen $(n=19)$, or the foramen recently closed $(n=10)$ are shown in Table 1. Individuals closing their apical foramen appear from the first to the second autumn, ie, from 6 to 18 months of age.

Table 1. Specimens of the sample showing any stage of closure of apical foramen.

\begin{tabular}{lccc}
\hline Season & Open foramen & Closing foramen & Recently closed foramen \\
\hline 1st autumn & 2 & 8 & 0 \\
1st winter & 3 & 5 & 1 \\
2nd spring & 1 & 3 & 2 \\
2nd summer & 0 & 1 & 0 \\
2nd autumn & 0 & 2 & 4 \\
2nd winter & 0 & 0 & 3 \\
Total & 6 & 19 & 10 \\
\hline
\end{tabular}

\section{Period of line formation}

A total of 108 specimens of the sample had a dark line developing in the surface of the root (Table 2), just under the periodontal membrane. The month including the highest number of specimens with a line under formation was January $(n=24)$. Significant differences were found when comparing number of specimens from spring-summer versus autumn-winter $\left(n=108, \chi^{2}=5.034, \mathrm{df}=1, p=0.025\right)$, indicating that lines are usually formed in autumn-winter.

Table 2. Observed and expected values for each seasonal period, related to the number of specimens showing a line under formation in the canine root. Expected values for the test were obtained by calculating specimens informative for the variable in proportion to the total sample for each seasonal interval.

\begin{tabular}{lccc}
\hline Season & Total sample & Observed & Expected \\
\hline Autumn-winter & 136 & 98 & 89 \\
Spring-summer & 29 & 10 & 19 \\
Total & 165 & 108 & 108 \\
\hline
\end{tabular}

\section{Formation of the first dark line}

Out of 25 specimens from the first year (kittens), 13 had no line formed and 12 had a non-continuous line (Fig. 1A). Out of 46 specimens from the second year (yearlings), 26 had just one continuous line and 20 had two lines. Among the latter 20 specimens, the outer line was always continuous, but the inner line could be 

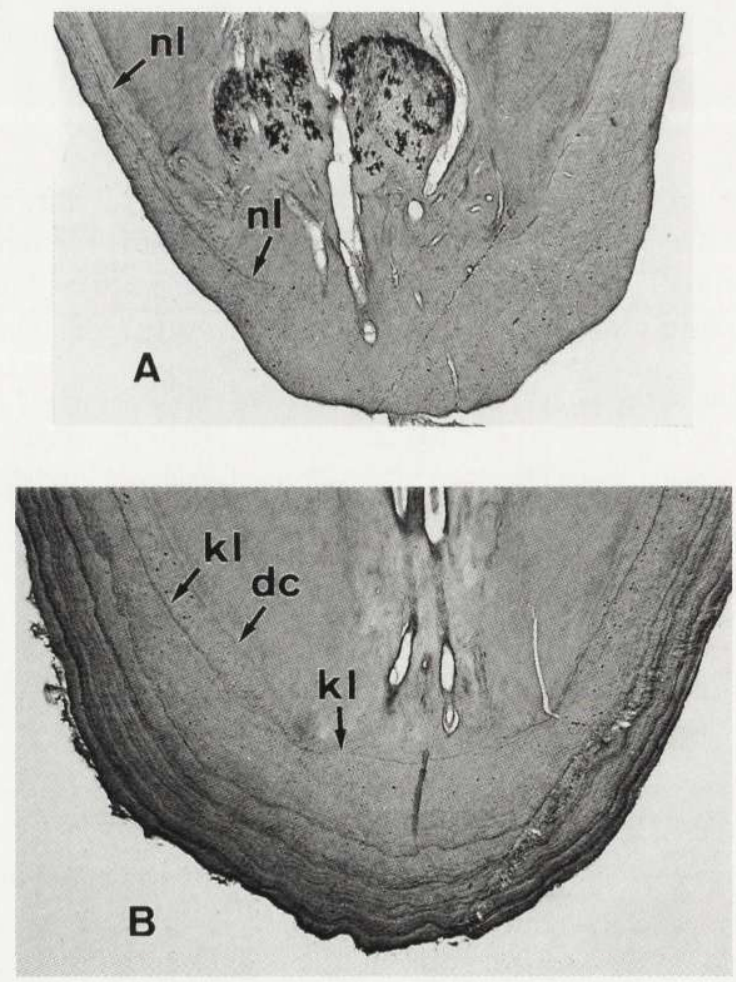

Fig. 1. A - an individual 19 months old showing a non-continuous line (nl) formed ( $20 \times$ magnification). $\mathrm{B}$ - an individual 6 years 9 months old showing a kitten line $(\mathrm{kl})$ and several multiple lines (20x magnification). dc - dentin-cementum junction.

either non-continuous ( $n=14$ ) or continuous $(n=6)$. When that internal line was continuous, it was too close to the dentin-cementum junction (Fig. 1B), and could be well differentiated from the other continuous lines in older specimens. We have called it "kitten line" (Fig. 1B). In the whole sample, 50.9\% of specimens show a non-continuous line and $6.7 \%$ show a kitten line. The remainder $42.4 \%$ show neither non-continuous nor kitten line.

\section{Counting the number of lines}

The number of lines other than non-continuous and kitten is easy to count when they are simple and appear in a small number. However, we have found in some specimens "multiple lines" (Fig. 2), which sometimes are difficult to differentiate (see Material and methods). Identification of individual lines was also difficult in old animals, since lines tend to concentrate in outer layers. Also, identification of lines forming at the periphery of the root was made carefully because they can be mistaken with the periodontal membrane. The oldest specimen of the sample had 10 continuous lines. 


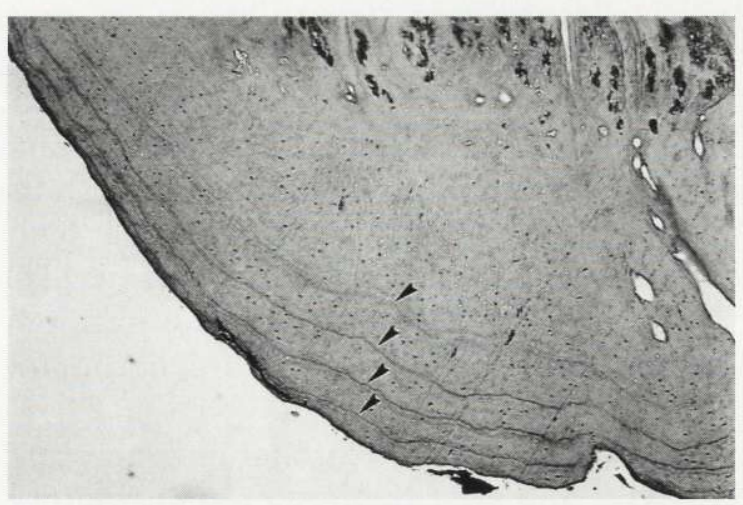

Fig. 2. An individual 5 years 6 months old showing 4 continuous, double lines (40× magnification). Observed at $100 \times$, it shows 1 more line under formation.

\section{Age estimation}

Information provided above is enough to estimate the age in years of Iberian wildcats. However, for a most accurate estimation, we suggest to consider 3 parameters, as proposed by García-Perea (1991) for Lynx pardinus: (1) months from birth to the period of line formation, (2) number of continuous lines, and (3) months from the last line formed to death (see Discussion).

\section{Discussion}

Assuming that wildcats are born in April, and permanent canines erupt when they are 6-7 months old (Condé and Schauenberg 1978), kittens should wear milk dentition during their first summer and acquire their permanent canines in October-November. After Grue and Jensen (1979), cementum development is usually initiated just at the time of the canine eruption, ie, in October-November. Our data confirm that closure of apical foramen starts in the first October, continuing through winter and spring. We have no data for the second summer, but 2 specimens from the second autumn showing a closing foramen, and 7 specimens from autumn-winter with the foramen recently closed indicate that the closure process continues through summer and autumn. Based on this information, we may conclude that closure of apical foramen occurs during the age of 6 to 18 months. This period is somewhat longer than that reported for lynxes, 12-19 months (Saunders 1964, Johnson et al. 1981, Kvam 1984, Zapata et al. 1997).

The period for line formation found in our sample, autumn-winter, is similar to that reported for other carnivores from temperate regions, eg Lynx pardinus (Zapata et al. 1997), Mustela erminea, from Denmark (Grue and Jensen 1979), Vulpes vulpes from southern Spain (Zapata et al. 1995). 
From our results related to the formation of the first dark line, we conclude that during the first autumn-winter wildcats may produce either a non-continuous line, or a kitten line, or no line. The presence of non-continuous and kitten lines is linked to the timing of closure of apical foramen (Grue and Jensen 1979). This is so because the period of line formation overlaps completely with the period of closure of the foramen. The type of line formed during the first year would depend on individual variation for the timing of foramen closure. If apical foramen is closed in spring-summer, the line formed in the preceeding autumn-winter would be non-continuous. However, if the foramen is closed in autumn-winter, it would be possible to find a continuous kitten line. The fact that $50.9 \%$ of specimens show a non-continuous line and only $6.7 \%$ show a kitten line suggests that most likely the closure of apical foramen occurs after the first autumn-winter. This is consistent with the timing estimated above, although it suggests that the closure period concentrates from 12 to 18 months of age. Non-continuous lines formed during the first year have been found in other felids, eg Lynx lynx (Kvam 1984) and Lynx pardinus (García-Perea 1991). We have found no references about kitten lines, although Grue and Jensen (1979) mention the possibility of formation of more or less continuous lines in the first year of life of individuals of $L$. lynx from Norway, and Canis latrans, from Canada, under similar circumstances to those found in Iberian wildcats. Considering that the month including more wildcats forming lines is January, we have estimated that non-continuous and kitten lines are formed in that month, when specimens are 9 months old.

Since lines formed during the first year may appear in a variable proportion of the sample, it is advisable to count the lines formed during and after the second autumn-winter, as proposed by Grue and Jensen (1979), Kvam (1984) and other authors. The first of these lines would be formed when wildcats are 21 months old (taking again January as the month of formation). For this reason, age of Iberian wildcats can be estimated in years by adding 1 to the number of continuous lines, being careful with identification of potential kitten lines.

Looking for a more accurate age estimation, we have calculated the 3 parameters proposed by García-Perea (1991). Months from birth to the formation of the first line is a figure of invariable value, 9 months (April to January). The number of continuous (non kitten) lines can be determined after solving the identification problems mentioned under Results. Months from the formation of the last continuous line to death is always a positive figure, except for specimens dead before January and showing a line under formation, in which case it is a negative figure. Based on these 3 parameters, we have established a formula for age estimation that can be expressed as follows, $A=9 m+L y \pm M m$ where $A$ is absolute age, $9 \mathrm{~m}$ is 9 months, Ly is the number of continuous lines corresponding to years of life, and $\mathrm{Mm}$ is the number of months from the last January to the month of death. Birth and death parameters are very important for an accurate age estimation, because by ignoring them we can make an error of up to 1 year, and that is an important error for specimens between 1 and 3 years old. 
Applying this formula to the individual with 10 continuous lines, dead in January, we have estimated an age of 10 years and 9 months $(A=9$ months +10 years +0 ). These data suggest that the lifespan of Iberian wildcats could be around 11 years, but more information is warranted to confirm this hypothesis.

Acknowledgements: We thank J. Gisbert, J. Cabot, Solís Fernández, M. Meijide, J. Barreiro, J. M. Gil, G. Valenzuela, N. Guzmán and J. Jiménez for providing the materials examined for this study. J. Gisbert and R. Fernández-Salvador cooperated in different stages of the study. J. Martín, P. López, R. Gómez-Calmaestra, and E. Moreno gave useful suggestions about the manuscript. J. Gisbert and

M. Alcobendas helped with the figures. Financial support was partially provided by DGICYT project PB95-0235 and by a predoctoral fellowship of the Consejo Superior de Investigaciones Científicas Museo Nacional de Ciencias Naturales to RB. Part of this study was developed thanks to the logistical support of "El Ventorrillo" field station (MNCN).

\section{References}

Condé B. and Schauenberg P. 1978. Remplacement des canines chez le Chat forestier Felis silvestris Schreb. Revue suisse de Zoologie 85: 241-245.

Crowe D. M. 1972. The presence of annuli in bobcat tooth cementum layers. The Journal of Wildlife Management 36: 1330-1332.

García-Perea R. 1991. Variabilidad morfológica del género Lynx Kerr, 1792 (Carnivora: Felidae). Complutense University, Madrid: 1-555.

García-Perea R., Baquero R. A., Fernández-Salvador R. and Gisbert J. 1996. Desarrollo juvenil del cráneo en las poblaciones ibéricas de Gato Montés, Felis silvestris Schreber, 1775. Doñana Acta Vertebrata 23: 153-164.

Grue H. and Jensen B. 1979. Review of formation of incremental lines in tooth cementum of terrestrial mammals. Danish Review of Game Biology 11: 1-48.

Johnson N. F., Brown B. A. and Bosomworth J. C. 1981. Age and sex characteristics of bobcat canines and their use in population assessment. Wildlife Society Bulletin 9: 203-206.

Klevezal G. A. and Kleinenberg S. E. 1967. Age determination of mammals from annual layers in teeth and bones. Israel Program for Scientific Translations, 1969, Jerusalem: 1-128.

Kvam T. 1984. Age determination in the European lynx (Lynx lynx) by incremental lines in canine tooth cementum. Acta Zoologica Fennica 171: 221-223.

Morris P. 1972. A review of mammalian age determination methods. Mammal Review 2: 69-104

Reimers E. and Nordby O. 1968. Relationship between age and tooth cementum layers in Norwegian Reindeer. The Journal of Wildlife Management 32: 957-961.

Saunders J. K. 1964. Physical characteristics of the Newfoundland lynx. Journal of Mammalogy 45: 36-47.

Schauenberg P. 1969. L'identification du Chat forestier d'Europe Felis s. silvestris Schreber, 1777 par une méthode ostéométrique. Revue suisse de Zoologie 76: 433-441.

Schauenberg P. 1974. Le chat sauvage démystifié. Musées de Genève 148: 2-6.

Stahl O., Artois M. and Aubert M. F. A. 1988. Organisation spatiale et déplacements des chats forestiers adultes (Felis silvestris, Schreber, 1777) en Lorraine. Revue d'Ecologie (Terre Vie) 47: $113-132$.

Zapata S., Travaini A. and Delibes M. 1995. Comparación entre varias técnicas de estimación de la edad en zorros, Vulpes vulpes de Doñana (Sur de la Península Ibérica). Doñana Acta Vertebrata 22: 29-50.

Zapata S., García-Perea R., Beltrán J. F., Ferreras P. and Delibes M. 1997. Age determination of Iberian lynx (Lynx pardinus) using canine radiograph and cementum annuli enumeration. Zeitschrift für Säugetierkunde 62: 119-123.

Received 5 May 1998, accepted 11 January 1999 